'Hospital de Loncoche, Loncoche, Chile.

${ }^{2}$ Departamento de Consultas y Procedimientos. Hospital Clínico de la Universidad de Chile. Santiago, Chile.

anterno de la Carrera de

Medicina. Escuela de Medicina. Universidad Diego Portales. Santiago, Chile.

${ }^{b} \mathrm{MSc}$ en Bioestadística, MBA, MSc en Políticas Públicas.

Este estudio fue realizado sin fuente de apoyo financiero.

Recibido el 29 de febrero de 2016, aceptado el 29 de septiembre de 2016.

Correspondencia a: Juan Haase juan.haase@mail.udp.cl

\section{Determinantes sociales de la edad de fallecimiento por causa cardiovascular}

\author{
JUAN HAASEa , SEBASTIÁN LAVANDEROS ${ }^{1}$, \\ CAMILO RIQUELMEa ${ }^{\mathrm{a}}$, ANDREA MORALES ${ }^{2, \mathrm{~b}}$
}

\section{Association of socio-demographic factors with the age at death due to cardiovascular diseases}

Background: The association of cardiovascular diseases with socio-demographic factors has not been fully explored. Aim: To analyze the association of socio-demographic features with the survival time of individuals who died due to cardiovascular diseases. Material and Methods: The death registries published by the Ministry of Health were analyzed. All deaths due to cardiovascular diseases occurred between 2001 and 2013 in people aged over one year were considered. Using a principal component analysis, the age at death was associated with socio-demographicfeatures such as sex, marital status, residence zone, schooling, work status and medical care prior to death. Results: A total of 293,370 cardiovascular deaths were analyzed. The median age at death was 77 years. Not receiving medical care prior to death, was significantly associated with a lower age at the moment of the decease, mainly between 20 and 80 years of age. Among men, being occupationally active (hazard ratio ( $H R=1.5$ $p<0.01)$ and being unemployed $(H R=1.1 p<0.01)$ were risks factors for early cardiovascular death. Being married, having higher schooling, residing in urban areas and having prior medical care were associated with a higher age at the moment of decease. Conclusions: Socio-demographic profiles are associated with the age at death due to cardiovascular diseases. The effect of not receiving medical care on the age at death is noteworthy and reflects social inequities in the access to health care.

(Rev Med Chile 2016; 144: 1464-1472)

Key words: Cardiovascular Diseases; Mortality; Socioeconomic Factors; Demography; Survival.

\section{L} as enfermedades cardiovasculares (ECV) constituyen la principal causa de muerte en Chile, con $27,1 \%$ de todas las defunciones el año 2011 y una tasa bruta de mortalidad que ha fluctuado entre 142 y 159 por 100 mil habitantes entre los años 2000 a $2011^{1,2}$. Este perfil epidemiológico, reflejo del envejecimiento paulatino de la población, replica la realidad sanitaria de países desarrollados o en vías avanzadas de desarrollo ${ }^{3,4}$.

Mientras que la asociación a factores clínicos, como Diabetes Mellitus o Hipertensión Arterial, y el riesgo de desarrollar una ECV está ampliamente documentada en la literatura ${ }^{5-8}$, las evidencias sobre su relación a factores sociodemográficos son escasas.

A nivel internacional, se han reportado evidencias que relacionan el desarrollo de ECV con edad mayor a 55 años, hogares de jefatura masculina, residencia urbana ${ }^{9}$, menor nivel educacional ${ }^{10} \mathrm{y}$ de ingresos ${ }^{11}$. En Chile algunos trabajos han documentado mayor mortalidad general o mayor tasa de Años de Vida Potencialmente Perdidos a menor ingreso y/o baja escolaridad ${ }^{12-14}$. En relación a mortalidad por ECV, en nuestro medio se han 
reportado algunas relaciones entre causas específicas de ECV (cardiopatía isquémica e insuficiencia cardíaca descompensada) con factores sociodemográficos como edad avanzada, estado civil soltero, ruralidad o bajo nivel sociocultural ${ }^{15,16}$. Sin embargo, estos estudios fueron realizados en grupos específicos de la población nacional, en patologías específicas y/o en un tiempo de análisis acotado, en algunos casos a un año. A la fecha no existen estudios basados en estadísticas nacionales que exploren la asociación de mortalidad por ECV con factores socioeconómicos y demográficos, lo cual resulta fundamental para el entendimiento de los factores de riesgo "no clínicos" de estas patologías, tal como se ha mostrado a nivel internacional ${ }^{17-21}$.

En este estudio proponemos un análisis multivariante de la interacción de factores socioeconómicos y demográficos asociados a la edad de fallecimiento por mortalidad cardiovascular, considerando el total de sujetos fallecidos en Chile en los últimos 13 años con datos disponibles. Esto contribuirá a un futuro análisis de desigualdad de acceso y resultados, que a su vez aportaría al diseño de políticas públicas efectivas que busquen intervenir la principal causa de mortalidad en Chile y el mundo.

\section{Metodología}

Se realizó un estudio analítico de la sobrevida por mortalidad cardiovascular del universo de defunciones en mayores de 1 año, ocurridas en Chile entre los años 2001 y 2013, a partir de la información disponible en las bases de datos de defunciones, publicadas por el Departamento de Estadísticas e Información de Salud (DEIS) del Ministerio de Salud ${ }^{2}$.

Como mortalidad de causa cardiovascular se consideraron, según la décima versión de la Clasificación Internacional de Enfermedades (CIE-10), los códigos que pudieran ser agrupados en las siguientes causas de fallecimiento: enfermedad cerebrovascular (I600-I679), patología aneurismática (I710-I729), hipertensiva (I100-I119 y I130-I139), tromboembolismo pulmonar y patología trombótica periférica (I260-I279), enfermedad ateroembólica periférica (I700-I709 y I740-I749), patologías de origen cardíaco valvular reumática (I00-I099) y no reumática (I330-I398), isquémica (I200-I259), del pericardio (I300-I328), del miocardio (I400-I438), insuficiencia car- díaca (I500-I509), del sistema excito-conductor (I440-I459 y I470-I499) y otras causas cardíacas $(\mathrm{I} 510-\mathrm{I} 519)^{22}$.

De los registros de defunciones, se consideraron como características demográficas y socio-económicas: sexo, estado civil, residencia urbana o rural, escolaridad (calculada en número de años cursados, en base al último curso y nivel de instrucción alcanzados) y condición de actividad laboral (inactivo, activo o cesante). Las variables categóricas estado civil y actividad laboral fueron ingresadas a los análisis previa construcción de variables indicadores o dummy. Además, se incluyó si el paciente recibió atención médica previa al fallecimiento.

Se realizó un análisis univariado de sobrevida por regresión de Cox, estimando Hazard ratios (HR) para identificar posibles factores predictores de la edad de fallecimiento. Considerando que estas variables presentan multi colinealidad al incluirlas en una regresión multivariante, se realizó un análisis de componentes principales para la identificación de perfiles de los fallecidos. Esta es una técnica estadística reduccionista utilizada ante la presencia de información redundante (generada por altas correlaciones, o multi colinealidad, entre un grupo de variables), que busca analizar y explicar estas interacciones, construyendo un número menor de nuevas variables no correlacionadas entre sí (que no tengan repetición o redundancia en la información), que explicarán gran parte de la variabilidad total. Estas nuevas variables, llamadas conjunto de componentes principales, son combinaciones lineales de las anteriores que se van construyendo según el orden de importancia en cuanto a la variabilidad total que recojan de la muestra. De éstos, se deben seleccionar aquellos componentes con valores propios (eigenvalues) mayores a 1, que representan los escalares asociados a los vectores de transformación lineal. Una vez seleccionados los componentes principales, se representan en forma de matriz, donde cada celda corresponde a la correlación entre la variable original y el componente principal. Las mayores correlaciones permitirán la identificación de cada perfil o componente (por consenso, correlaciones mayores a 0,3$)^{23-24}$.

Finalmente, se evaluó la significancia de los perfiles construidos como predictores de la edad de fallecimiento por mortalidad cardiovascular, a través de regresión de Cox multivariante. 
El análisis de datos se realizó con programa Stata ${ }^{\circledR} 12.0$ (licenciado para un autor).

Se utilizaron bases de datos públicas, disponibles en la web del DEIS, que no consideran datos identificatorios de los casos, por lo cual no se requirió aprobación por Comité de ética previo al desarrollo de este estudio.

\section{Resultados}

Se analizaron 293.370 defunciones por mortalidad cardiovascular en Chile, entre los años 2001 y 2013 , que correspondieron a $25,6 \%$ del total de muertes en mayores de 1 año. Se obtuvo una mediana de edad de fallecimiento de 77 años. Las características sociodemográficas y la distribución relativa de las causas específicas de mortalidad cardiovascular se presentan en la Tabla 1; en tanto, la Tabla 2 evidencia la distribución de estas causas por grupos etarios y la Tabla 3, la edad de fallecimiento según causa.

El análisis de regresión univariado, mostró una edad significativamente menor al fallecimiento en: sexo masculino (74 vs 81 años en mujeres; $\mathrm{HR}=1,65$, IC: $1,640-1,664)$, divorciados (63 vs 77 años en casados; HR = 2,80, IC: 2,523-3,098), activos laboralmente (56 vs 79 años en inactivos; $\mathrm{HR}=6,30$, IC: 6,220-6,379) o cesantes (53 años; $\mathrm{HR}=14,51$ IC: $13,971-15,069)$. Asimismo, la escolaridad se asoció débilmente a edades menores de fallecimiento ( $\mathrm{HR}=1,03$, IC: 1,032-1,034). En tanto, ser viudo se asoció a mayores edades (81 años; HR = 0,83, IC: 0,819-0,845). Sin embargo, el análisis de estas características presenta endogeneidad, puesto que variables como estado civil, escolaridad y actividad laboral, están fuertemente determinadas por la cohorte generacional de un sujeto y su edad de fallecimiento.

Por otra parte, no recibir atención médica previa resultó significativamente asociado a menor edad de fallecimiento ( 73 vs 79 años; $H R=1,28$, IC: 1,264-1,298), asociación que se mantuvo entre los 20 y 80 años al analizarlo por grupos etarios $(\mathrm{p}<0,001)$; en tanto, la asociación se invierte en edades menores (Figura 1).

Al evaluar la correlación entre estos factores sociodemográficos, se obtuvieron algunas relaciones significativas entre variables como: sexo masculino y escolaridad ( $\mathrm{rho}=0,1149 ; \mathrm{p}<0,001)$ o estar laboralmente activo $(\mathrm{rho}=0,2658 ; \mathrm{p}<0,001) \mathrm{y}$
Tabla 1. Características sociodemográficas y causas de muerte en fallecidos por enfermedad cardiovascular. Chile, 2001-2013

\begin{tabular}{|c|c|}
\hline & Mediana \\
\hline Variables continuas & (RI / Rango) \\
\hline Edad & $77(67-85$ / 0-119) \\
\hline Escolaridad (total) & $6(4-9$ / 0-19) \\
\hline Escolaridad en mayores de 20 años & $6(4-9 / 0-19)$ \\
\hline Variables categóricas & $\% \quad(n)$ \\
\hline \multicolumn{2}{|l|}{ Sexo } \\
\hline Masculino & $51,11(149.950)$ \\
\hline Femenino & $48,89(143.420)$ \\
\hline \multicolumn{2}{|l|}{ Estado civil } \\
\hline Soltero & $60,65(177.572)$ \\
\hline Casado & $33,40 \quad(978)$ \\
\hline Viudo & $5,82 \quad(17.054)$ \\
\hline Divorciado $^{\dagger}$ & $0,12 \quad(365)$ \\
\hline \multicolumn{2}{|l|}{ Residencia } \\
\hline Urbana & $86,49(253.731)$ \\
\hline Rural & $13,51 \quad(39.639)$ \\
\hline \multicolumn{2}{|l|}{ Nivel de Instrucción } \\
\hline Analfabeto & $11,18 \quad(32793)$ \\
\hline Básica & $56,99(167.101)$ \\
\hline Media & $5,58 \quad(16.360)$ \\
\hline Secundaria & $21,81 \quad(63.959)$ \\
\hline Superior & $4,43 \quad(12.981)$ \\
\hline \multicolumn{2}{|l|}{ Actividad } \\
\hline Inactivos & $88,52(257.750)$ \\
\hline Activos & $10,49 \quad(30.559)$ \\
\hline Cesantes & $0,99 \quad(2.874)$ \\
\hline \multicolumn{2}{|l|}{ Grupo de causas de muerte } \\
\hline Isquémica & $34,65(101.645)$ \\
\hline Cerebrovascular & $28,92 \quad(84.853)$ \\
\hline Hipertensiva & $12,92 \quad(37.898)$ \\
\hline Insuficiencia cardíaca & $7,82 \quad(22.942)$ \\
\hline Arritmias & $4,98 \quad(14.607)$ \\
\hline Miocárdica & $2,76 \quad(8.104)$ \\
\hline Aneurismática & $(5.572)$ \\
\hline Trombótica (TEP-TVP) & $(4.871)$ \\
\hline Valvular & $(4.568)$ \\
\hline Reumática & $(2.721)$ \\
\hline Ateroembólica periférica & $(2.522)$ \\
\hline Pericárdica & $0,10 \quad(284)$ \\
\hline Otros & $0,95 \quad(2.783)$ \\
\hline
\end{tabular}

†Evidenciado sólo el año 2012. 
Tabla 2. Causas de muerte cardiovascular por grupos etarios. Chile, 2001-2013

\begin{tabular}{|c|c|c|c|}
\hline Grupo etario (años) & Causas de muerte & $\%$ & $\%$ acumulado \\
\hline \multirow{3}{*}{$\begin{array}{c}1-19 \\
(n=739)\end{array}$} & Cerebrovascular & $35,45 \quad(262)$ & 35,45 \\
\hline & Miocárdica & 25,30 & 60,76 \\
\hline & Otros & 8,66 & 69,42 \\
\hline \multirow{3}{*}{$\begin{array}{c}20-39 \\
(n=4.777)\end{array}$} & Cerebrovascular & $33,91 \quad(1.620)$ & 33,91 \\
\hline & Isquémica & $24,47 \quad(1.169)$ & 58,38 \\
\hline & Miocárdica & $9,29 \quad(444)$ & 67,68 \\
\hline \multirow{3}{*}{$\begin{array}{c}40-59 \\
(n=36.205)\end{array}$} & Isquémica & $40,94(14.823)$ & 40,94 \\
\hline & Cerebrovascular & $30,87(11.177)$ & 71,81 \\
\hline & Hipertensiva & $5,57 \quad(2.016)$ & 77,38 \\
\hline \multirow{3}{*}{$\begin{array}{c}60-79 \\
(n=124.349)\end{array}$} & Isquémica & $38,95(48.429)$ & 38,95 \\
\hline & Cerebrovascular & $29,80(37.057)$ & 68,75 \\
\hline & Hipertensiva & $10,03(12.469)$ & 78,77 \\
\hline \multirow{3}{*}{$\begin{array}{c}\geq 80 \\
(n=127.300)\end{array}$} & Isquémica & $29,22(37.193)$ & 29,22 \\
\hline & Cerebrovascular & $27,29(34.737)$ & 56,50 \\
\hline & Hipertensiva & $18,27(23.261)$ & 74,78 \\
\hline
\end{tabular}

Tabla 3. Edad de fallecimiento por enfermedad cardiovascular según causas. Chile, 2001-2013

\begin{tabular}{|lccc|}
\hline Grupo de causas de muerte & Mediana (RI) & HR & IC \\
\hline Isquémica $^{\dagger}$ & $75(65-83)$ & - & - \\
\hline Cerebrovascular & $77(66-84)$ & 0,948 & $0,940-0,957$ \\
\hline Hipertensiva & $83(75-89)$ & 0,631 & $0,624-0,639$ \\
\hline Insuficiencia cardíaca & $83(74-89)$ & 0,630 & $0,621-0,639$ \\
\hline Arritmias & $82(74-88)$ & 0,678 & $0,666-0,689$ \\
\hline Miocárdica & $70(57-80)$ & 1,414 & $1,383-1,447$ \\
\hline Aneurismática & $72(62-80)$ & 1,346 & $1.310-1.382$ \\
\hline Trombótica (TEP-TVP) & $73(59-83)$ & 1,110 & $1,078-1,142$ \\
\hline Valvular & $72(60-81)$ & 1,219 & $1,184-1,256$ \\
\hline Reumática & $66(55-76)$ & 1,766 & $1,700-1,834$ \\
\hline Ateroembólica periférica & $84(77-91)$ & 0,541 & $0,520-0,563$ \\
\hline Pericárdica & $60(45-71)$ & 2,637 & $2,347-2,963$ \\
\hline Otros & $67(51-80)$ & 1,302 & $1,254-1,352$ \\
\hline
\end{tabular}

†La causa isquémica se utilizó como categoría de referencia.

residencia urbana y escolaridad (rho $=0,2630$; $\mathrm{p}<0,001)$. Para ponderar este efecto de multicolinealidad, se realizó un análisis de componentes principales, determinando 5 perfiles de sujetos en cuanto al conjunto de características sociodemográficas, que explican en conjunto $75,1 \%$ de la varianza total (Figura 2 y Tabla 4$)$ :
- 1: hombres laboralmente activos.

- 2: estado civil casado.

- 3: mayor escolaridad, residencia urbana y atención médica previa.

- 4: viudos.

- 5: cesantes. 
A.

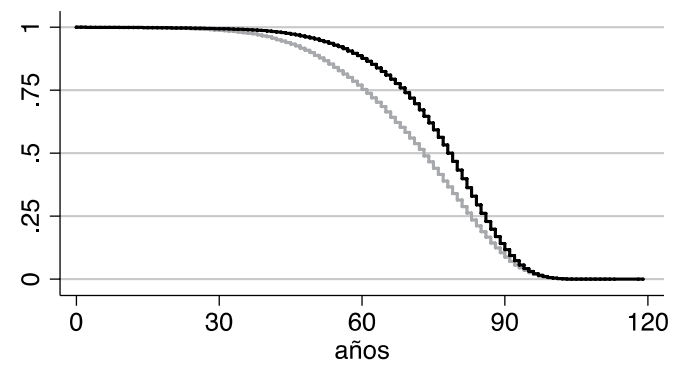

C.

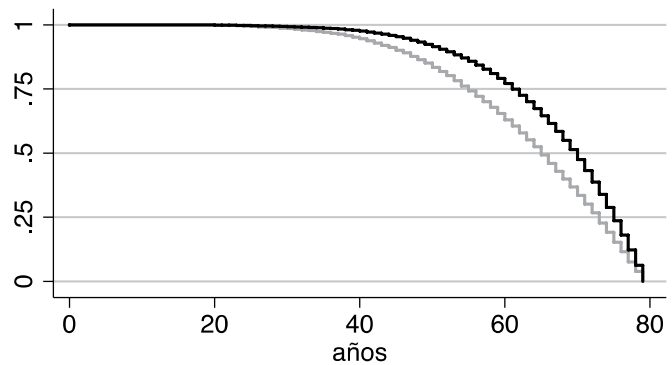

Sin atención médica
B.

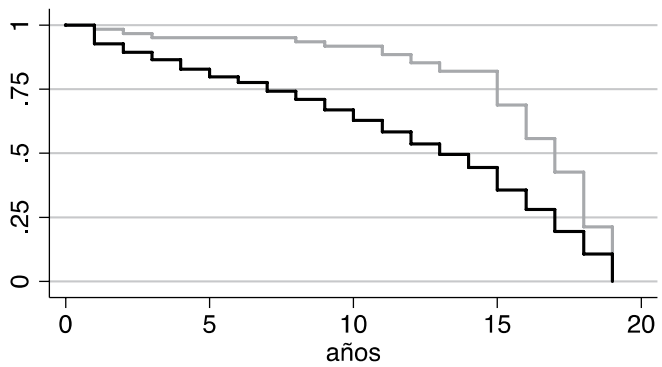

D.

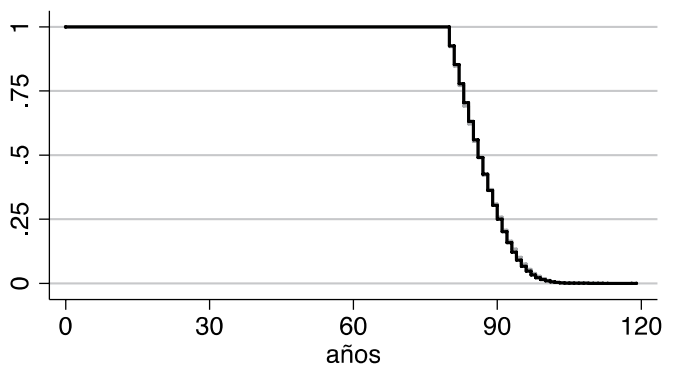

Con atención médica

Figura 1. Edad de fallecimiento por muerte cardiovascular según atención médica previa. Chile, 2001-2013. A. Universo de fallecidos. B. Fallecidos menores de 20 años. C. Fallecidos entre 20 y 80 años. D. Fallecidos mayores de 80 años.

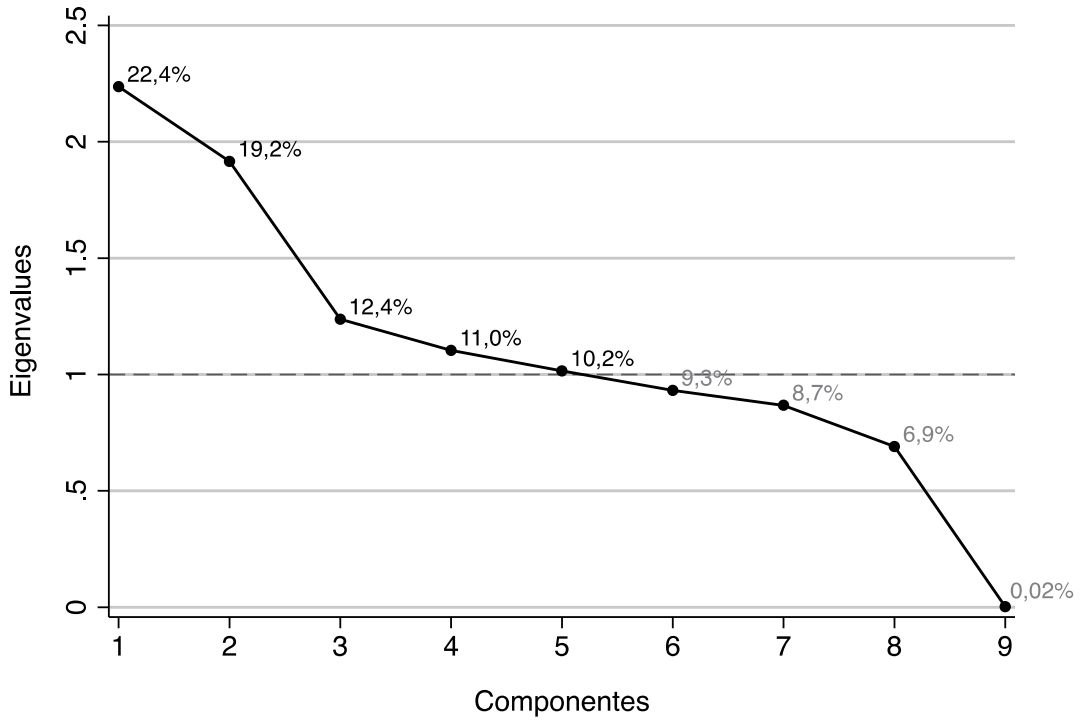

Figura 2. Selección de componentes principales para determinación de perfiles sociodemográficos. Se seleccionaron aquellos componentes con eigenvalues $>1$ (componentes 1-5). 
Tabla 4. Matriz de correlación de componentes principales para determinación de perfiles sociodemográficos

\begin{tabular}{|lccccc|}
\hline Variables & $\mathbf{1}$ & $\mathbf{2}$ & $\mathbf{P e r f i l e s}$ & $\mathbf{4}$ & $\mathbf{5}$ \\
\hline Hombres & $\mathbf{0 , 3 0 7 8}$ & 0,0118 & $-0,158$ & $-0,0975$ & 0,085 \\
\hline Escolaridad & 0,2682 & 0,1103 & $\mathbf{0 , 5 4 2 3}$ & $-0,0679$ & $-0,0147$ \\
\hline Soltero & $-0,0226$ & $-0,6735$ & 0,083 & $-0,3252$ & $-0,0562$ \\
\hline Casado & 0,0683 & $\mathbf{0 , 7 0 0 5}$ & $-0,1432$ & $-0,1268$ & 0,0058 \\
\hline Viudo & $-0,0991$ & $-0,1047$ & 0,1291 & $\mathbf{0 , 9 0 6 5}$ & 0,1007 \\
\hline R. urbana & 0,0119 & 0,1024 & $\mathbf{0 , 7 0 8 8}$ & $-0,0843$ & 0,0063 \\
\hline Inactivo & $-0,6357$ & 0,0758 & 0,0273 & $-0,1051$ & 0,0067 \\
\hline Activo & $\mathbf{0 , 6 1 0 4}$ & $-0,0669$ & $-0,0454$ & 0,1315 & $-0,2938$ \\
\hline Cesante & 0,1612 & $-0,0385$ & 0,0554 & $-0,0727$ & $\mathbf{0 , 9 3 2 1}$ \\
A. Médica & $-0,1247$ & 0,1011 & $\mathbf{0 , 3 5 8 7}$ & $-0,0396$ & $-0,1552$ \\
\hline
\end{tabular}

Para cada perfil, se consideraron las correlaciones con valores mayores a 0,3.

Tabla 5. Tiempo de sobrevida por muerte cardiovascular según perfiles sociodemográficos. Chile, 2001-2013

\begin{tabular}{|lccc|}
\hline Perfiles & HR & p value & IC \\
\hline Hombres laboralmente activos & 1,471 & 0,000 & $1,467-1,475$ \\
\hline Casados & 0,961 & 0,000 & $0,959-0,964$ \\
\hline Mayor escolaridad, residencia urbana y atención médica previa & 0,973 & 0,000 & $0,970-0,976$ \\
Viudos & 1,003 & 0,068 & $1,000-1,007$ \\
\hline Cesantes & 1,093 & 0,000 & $1,089-1,097$ \\
\hline Observaciones & 266.257 & & \\
\hline
\end{tabular}

A continuación se evaluó un modelo de sobrevida multivariado para estos perfiles, evidenciando que: i) el perfil 1 (hombres laboralmente activos) y 5 (cesantes), tendrían 1,47 [IC: $1,467-1,475$ ] y 1,09 [IC: $1,089-1.097$ ] veces más riesgo de fallecer por mortalidad cardíaca a edades más precoces, respectivamente; mientras que, sujetos con perfil 2 (casados) y 3 (mayor escolaridad, residencia urbana y atención médica previa) presentarían 0,96 [IC: 0,959-0,964] y 0,97 [IC: $0,970-0,977$ ] veces menos riesgo de mortalidad cardíaca a edades tempranas. Ser viudo no resultó significativo como perfil de riesgo (Tabla 5).

\section{Discusión}

Se estima que para el año 2030 las ECV continuarán representando la primera causa de muerte en el mundo, proyectándose un aumento sustancial en su prevalencia y en los costos derivados, principalmente, de aquellos atribuibles a la pérdida de productividad ${ }^{25,26}$.

A la fecha, la gran mayoría de los esfuerzos en investigación han estado enfocados en la identificación, modificación y tratamiento de sus factores de riesgo clínicos. Sin embargo, a pesar de los grandes avances alcanzados en esta senda, poco se ha explorado sobre la identificación de factores 
socioeconómicos en el desarrollo de estas patologías, que podrían ser consideradas las "causas de las causas" de las ECV y que, a su vez, pudiesen provocar desigualdades sociodemográficas en su distribución poblacional.

Como una contribución en este sentido, presentamos una descripción del universo de muertes por causa cardiovascular en Chile a lo largo de 13 años y un análisis de las asociaciones existentes entre la edad de fallecimiento y algunos factores sociodemográficos. El universo estudiado reflejó un predominio de sexo masculino, estado civil casado, inactividad laboral, residencia urbana y baja escolaridad, esta última con una mediana de 6 años, la cual no estuvo influenciada por los fallecidos de menores edades (al observarse esta misma distribución en mayores de 20 años).

En relación a las etiologías, 76,5\% de las muertes por ECV correspondieron a las causas isquémica, cerebrovascular e hipertensiva, siguiendo este ordenamiento desde los 40 años. La causa cerebrovascular predominó en menores de 40 años (entorno a 35\%), lo cual resulta concordante con estudios que demuestran altas prevalencias de esta patología en este grupo, asociado a causas hematológicas, oncológicas, infecciosas, metabólicas y cardiopatías congénitas o adquiridas ${ }^{27,28}$.

Mediante análisis de componentes principales fue posible identificar cuatro perfiles sociodemográficos que, por análisis de sobrevida, resultaron significativamente asociados a la edad de fallecimiento por ECV; dos de estos perfiles estuvieron compuestos por más de una característica, reflejando la existencia de interacciones complejas entre estos factores, lo cual restringe el uso de un análisis de regresión directo.

En cuanto a los perfiles asociados a riesgo de fallecimiento a edades más tempranas: i) hombres activos laboralmente y ii) cesantes, es interesante el hecho de que en ambos pudiese subyacer el estrés como una causa biológicamente plausible, el cual podría estar vinculado tanto a tensión laboral excesiva en individuos activos, como a privación socioeconómica ante la condición de cesantía. Ambas conjeturas coinciden dentro de la denominada "teoría de la carga alostática", construida a partir de diversas investigaciones desde la década del '70 y que se ha relacionado a mayor riesgo cardiovascular ${ }^{29-30}$.

Por otra parte, el efecto protector de fallecimiento a edades más tardías encontrado en los otros dos perfiles: iii) estado civil casado y iv) sujetos con mayor educación, que habiten en zonas urbanas y accedan a atención médica previa al fallecimiento, concuerda también con investigaciones internacionales, en las cuales se ha reportado la influencia del estado civil, la educación y las inequidades socioeconómicas sobre la mortalidad general y cardiovascular ${ }^{17-21}$.

Asimismo, nuestros resultados concuerdan con otros reportes nacionales. Castro et al. analizaron el impacto de factores socioculturales en las características del cuidado post-hospitalario de pacientes ingresados por insuficiencia cardíaca descompensada en hospitales del registro ICARO, en el período 2006-2008. Los resultados mostraron que la edad mayor a 70 y un bajo nivel sociocultural (considerando nivel educacional, actividad laboral y previsión de salud) constituirían predictores de mortalidad, con HR de 2,17 y 1,57 respectivamente $^{15}$. Alonso et al. estudiaron la relación entre factores sociodemográficos y mortalidad por cardiopatía isquémica extrahospitalaria, a partir de los registros nacionales de defunciones entre 1997 y 2007, observando que la edad avanzada, el estado civil soltero y la ruralidad, constituían factores asociados a mortalidad extrahospitalaria en ambos sexos ${ }^{16}$.

Un resultado central de este estudio fue el hallazgo de que la atención médica previa al fallecimiento se asociara significativamente a mortalidad a edades más tardías, principalmente, en edades productivas y hasta los 80 años. Esto recalca la importancia del acceso a salud oportuno en patologías cardíacas. Específicamente en el contexto prehospitalario, existe evidencia que apoya el uso de desfibriladores de acceso público en Estados Unidos de Norteamérica, Europa e incluso Latinoamérica, estando ampliamente demostrado que la supervivencia a paros cardiorespiratorios extrahospitalarios mejora significativamente con la implementación de este recurso ${ }^{31-33}$.

En este sentido, el componente 3 encontrado en este estudio, al evidenciar la interacción entre residir en zonas urbanas, mayor educación y atención medica previa, revela la posibilidad de que una desigualdad de acceso a salud, generada por condiciones socioeconómicas, produzca una desigualdad de resultados en salud, en este caso la edad de fallecimiento por ECV.

Respecto a que en grupos etarios extremos (menores de 20 y mayores de 80 ) recibir atención 
médica no constituyera un factor protector, claramente, no debe ser interpretado como un riesgo de muerte precoz. Más bien se deben considerar posibles causas de endogeneidad en estos modelos: i) es altamente probable que estos grupos cuenten con atención previa al fallecimiento y ii) la limitada contribución de la acción médica a la supervivencia por un evento cardíaco, al considerar las causas de muerte más probables en estos grupos: patologías congénitas, en el caso edades menores y baja reserva fisiológica en los pacientes de mayor edad.

Sin duda, este estudio no está exento de limitaciones. Respecto de su diseño, se realizó un análisis de los fallecidos por causa cardiovascular, lo cual considera el tiempo de sobrevida como variable de interés y no el riesgo de fallecer por ECV, el cual requiere la construcción de tasas de supervivencia en relación al total de población expuesta. Sin embargo, un estudio de esta envergadura no es factible de realizar en la actualidad, puesto que no existen en Chile registros de sujetos con eventos cardiovasculares atendidos en sistemas públicos y privados; a la fecha, sólo se encuentran disponibles datos nacionales de egresos hospitalarios públicos (no individualizables) y de fallecimientos. Por otra parte, este análisis no considera el efecto de los factores clínicos clásicamente asociados a mortalidad cardiovascular, como diabetes mellitus o hipertensión arterial, dado que las bases de datos del DEIS registran sólo la causa directa de muerte proveniente de los certificados de defunción y no causas secundarias.

A pesar de estas limitaciones, este estudio, analizando el universo nacional de pacientes fallecidos por ECV, permite unificar, sistematizar y establecer fielmente las correlaciones esbozadas por otros trabajos, los cuales en relación a causas específicas de mortalidad por ECV, han utilizado un menor número de variables sociodemográficas, en algunos casos no considerando su interacción, en intervalos de tiempo más reducidos y/o a través de muestras del universo. Las asociaciones encontradas en este análisis entre la edad de fallecimiento por mortalidad cardiovascular y determinados perfiles sociodemográficos, apuntan a posibles desigualdades de acceso y resultados, destacando el riesgo de mortalidad precoz al no contar con atención médica previa. Estos hallazgos justifican la realización de futuros análisis de desigualdad, que contribuyan al apropiado diseño de políticas públicas sanitarias orientadas a la prevención de la principal causa de muerte en Chile.

\section{Referencias}

1. Ministerio de Salud de Chile, departamento de estadísticas e información de salud. Indicadores básicos de salud, Chile 2013. Disponible en: http://www.deis.cl/ wp-content/uploads/2013/12/ibs-2013.pdf [Consultado el 07 de enero de 2015].

2. Ministerio de Salud de Chile, departamento de estadísticas e información de salud. Mortalidad por causas, según sexo. Chile 2000-2011. Chile. Disponible en: http:// www.deis.cl/defunciones-y-mortalidad-por-causas/ [Consultado el 07 de enero de 2015].

3. Szot J. La Transición Demográfico-Epidemiológica en Chile, 1960-2001. Rev Esp Salud Pública 2003; 77: 605-13.

4. Valdivia G. Transición epidemiológica: la otra cara de la moneda. Rev Med Chile 2006; 134: 675-8.

5. Wilson P. Established risk factors and coronary artery disease: The Framingham Study. Am J Hypertens 1994; 7: 7S-19.

6. Wilson P, D'Agostino R, Levy D, Belanger A, Silbershatz H, Kannel W. Prediction of Coronary Heart Disease Using Risk Factor Categories. Circulation 1998; 97: 1837-47.

7. Yusuf S, Hawken S, Ounpuu S, Dans T, Avezum A, Lanas $\mathrm{F}$, et al. Effect of potentially modifiable risk factors associated with myocardial infarction in 52 countries (the interheart study): case-control study. Lancet 2004; 364: 937-52.

8. Jousilahti P, Vartiainen E, Tuomilehto J, Puska P. Sex, age, cardiovascular risk factors, and coronary heart disease: a prospective follow-up study of 14786 middle-aged men and women in finland. Circulation 1999; 99 (9): 1165-72.

9. Rahman M, Nakamura K, Seino K, Kizuki M. Sociodemographic factors and the risk of developing cardiovascular disease in Bangladesh. Am J Prev Med 2015; 48 (4): 456-61.

10. Borrell C, Azlor E, Rodríguez-Sanz M, Puigpino's R, Cano-Serral G, Pasarín M, et al. Trends in socioeconomic mortality inequalities in a southern European urban setting at the turn of the 21 st century. J Epidemiol Community Health 2008; 62: 258-66.

11. Lee D, Chiu M, Manuel D, Tu K, Wang X, Austin P, et al. Trends in risk factors for cardiovascular disease in Canada: temporal, socio-demographic and geographic factors for the Canadian Cardiovascular Outcomes Research Team. CMAJ 2009; 181: E55-66.

12. Sánchez H, Albala C. Desigualdades en salud: mortali- 
dad del adulto en comunas del Gran Santiago. Rev Med Chile 2004; 132: 453-60.

13. Sánchez H, Albala C, Lera L. Años de Vida Perdidos por muerte prematura (AVPP) en adultos del Gran Santiago Hemos ganado con Equidad. Rev Med Chile 2005; 133: 575-82.

14. Koch E, Romero T, Manríquez L, Paredes M, Ortúzar E, Taylor A, et al. Desigualdad educacional y socioeconómica como determinante de mortalidad en Chile: análisis de sobrevida en la cohorte del proyecto San Francisco. Rev Med Chile 2007; 135: 1370-9.

15. Castro P, Verdejo H, Garcés E, Concepción R, Sepúlveda $\mathrm{L}$, Lanas F, et al. Influencia de factores socioculturales en la evolución alejada de pacientes con Insuficiencia cardíaca. Rev Chil Cardiol 2009; 28: 51-62.

16. Alonso FT, Nazzal C, Alvarado ME. Mortalidad por cardiopatía isquémica en Chile: quiénes, cuántos y dónde. Rev Panam Salud Pública 2010; 28: 319-25.

17. Johnson N, Backlund E, Sorlie P, Loveless C. Marital status and mortality: the national longitudinal mortality study. Ann Epidemiol 2000; 10: 224-38.

18. Zajacova A. Education, gender, and mortality: Does schooling have the same effect on mortality for men and women in the US? Soc Sci Med 2006; 63: 2176-90.

19. Mackenbach JP, Cavelaars AE, Kunst AE, Groenhof F. Socioeconomic inequalities in cardiovascular disease mortality; an international study. Eur Heart J 2000; 21: 1141-51.

20. Page A, Lane A, Taylor R and Dobson A. Trends in socioeconomic inequalities in mortality from ischaemic heart disease and stroke in Australia, 1979-2006. Eur J Prev Cardiol 2012; 19: 1281-9.

21. Kamphuis CB, Turrell G, Giskes K, Mackenbach JP, van Lenthe FJ. Life course socioeconomic conditions, adulthood risk factors and cardiovascular mortality among men and women: a 17-year follow up of the GLOBE study. Int J Cardiol 2013; 168: 2207-13.

22. Paho.org [Internet]. Washington DC: PAHO; [actualizada en diciembre de 2013, consultado el 22 de agosto de 2016]. Disponible en: http://ais.paho.org/classifications/
Chapters/index.htm

23. Hair JF, Anderson RE, Tatham RL, Black WC. Análisis multivariante, $5^{\mathrm{a}}$ ed. Prentice Hall Iberia, Madrid, 1999. ISBN: 978-84-8322-035-1.

24. Martínez-González MA, Sánchez-Villegas A, Faulín Fajardo FJ. Bioestadística amigable, 2a ed. España, 2006. ISBN 84-7978-791-0.

25. Mathers C, Loncar D. Projections of Global Mortality and Burden of Disease from 2002 to 2030. PLoS Med. 2006; 3: e442. doi:10.1371/journal.pmed.0030442.

26. Mozaffarian D, Benjamin EJ, Go AS, Arnett DK, Blaha MJ, Cushman M, et al. Heart disease and stroke statistics-2015 update: a report from the American Heart Association. Circulation 2015; 131: e29-322.

27. Kamruzzaman AKM, Monzuara M, Haidary MH. Acute stroke in pediatric age group: a review. IBMC J 2011; 2: 28-32.

28. Ji R, Schwamm LH, Pervez MA, Singhal AB. Ischemic stroke and transient ischemic attack in young adults: Risk factors, diagnostic yield, neuroimaging, and thrombolysis. JAMA Neurol 2013; 70: 51-7.

29. Szanton S, Gill J, Allen J. Allostatic load: a mechanism of socioeconomic health disparities. Biol Res Nur 2005; 7: 7-15.

30. Seldenrijk A, Hamer M, Lahiri A, Penninx B, Steptoe A. Psychological distress, cortisol stress response and subclinical coronary calcification. Psychoneuroendocrinology 2012; 37: 48-55.

31. Hallstrom A, Ornato JP. Public-Access Defibrillation and Survival after Out-of-Hospital Cardiac Arrest. N Engl J Med 2004; 351: 637-46.

32. Agerskov M, Nielsen AM, Hansen CM, Hansen MB, Lippert FK, Wissenberg M, et al. Public Access Defibrillation: Great benefit and potential but infrequently used. Resuscitation 2015; 96: 53-8.

33. Niggemeyer A, Machado F, Xalambrí F, Albornoz H, Sánchez A, López P, et al. Resultados iniciales de la implementación de programas de acceso público a la desfibrilación en Uruguay. Rev Urug Cardiol [online] 2013; 28: 334-44. 